\title{
Morita Contexts for Corings and Equivalences*
}

\author{
Jawad Y. Abuhlail ${ }^{\dagger}$ \\ Mathematics Department, Birzeit University \\ Birzeit - Palestine
}

\begin{abstract}
In this note we study Morita contexts and Galois extensions for corings. For a coring $\mathcal{C}$ over a (not necessarily commutative) ground ring $A$ we give equivalent conditions for $\mathcal{M}^{\mathcal{C}}$ to satisfy the weak. resp. the strong structure theorem. We also characterize the so called cleft $C$-Galois extensions over commutative rings. Our approach is similar to that of Y. Doi and A. Masuoka in their work on (cleft) $H$-Galois extensions (e.g. Doi94, DM92]).
\end{abstract}

\section{Introduction}

Let $\mathcal{C}$ be a coring over a not necessarily commutative ring $A$ and assume $A$ to be a right $\mathcal{C}$-comodule through $\varrho_{A}: A \longrightarrow A \otimes_{A} \mathcal{C} \simeq \mathcal{C}, a \mapsto \mathrm{x} a$ for some group-like element $\mathbf{x} \in \mathcal{C}$ (see Brz02, Lemma 5.1]). In the first section we study from the viewpoint of Morita theory the relationship between $A$ and its subring of coinvariants $B:=A^{c o \mathcal{C}}:=\{b \in A \mid \varrho(b)=b \mathbf{x}\}$. We consider the $A$-ring ${ }^{*} \mathcal{C}:=\operatorname{Hom}_{A-}(\mathcal{C}, A)$ and its left ideal $Q:=\left\{q \in{ }^{*} \mathcal{C} \mid \sum c_{1} q\left(c_{2}\right)=\right.$ $q(c) \mathbf{x}$ for all $c \in \mathcal{C}\}$ and show that $B$ and ${ }^{*} \mathcal{C}$ are connected via a Morita context using ${ }_{B} A_{*_{\mathcal{C}}}$ and ${ }_{{ }^{C} \mathcal{C}} Q_{B}$ as connecting bimodules. Our Morita context is in fact a generalization of Doi's Morita context presented in Doi94.

In the second section we introduce the weak (resp. the strong) structure theorem for $\mathcal{M}^{\mathcal{C}}$. For the case ${ }_{A} \mathcal{C}$ is locally projective, in the sense of B. Zimmermann-Huignes, we characterize $A$ being a generator (a progenerator) in the category of right $\mathcal{C}$-comodules by $\mathcal{M}^{\mathcal{C}}$ satisfying the weak (resp. the strong) structure theorem. Here the notion of Galois corings introduced by T. Brzeziński [Brz02] plays an important role. The results and proofs are essentially module theoretic and similar to those of [MZ97] for the catgeory $\mathcal{M}(H)_{A}^{C}$ of Doi-Koppinen modules corresponding to a right-right Doi-Koppinen structure $(H, A, C)$ (see also [MSTW01] for the case $C=H$ ).

The notion of a $C$-Galois extension $A$ of a ring $B$ was introduced by T. Brzeziński and S. Majid in BM98, and is related to the so called entwining structures introduced in the

${ }^{*}$ MSC (2000): 16D90, 16S40, 16W30, 13B02

Keywords: Morita Contexts, Hopf Algebras, (Cleft) Galois Extensions, Entwining Structures, Entwined Modules.

${ }^{\dagger}$ Current Address: Department of Mathematical Sciences, Box \# 5046, KFUPM, 31261 Dhahran (Saudi Arabia), email: abuhlail@kfupm.edu.sa 
same paper. In the third section we give equivalent conditions for a $C$-Galois extension $A / B$ to be cleft. Our results generalize results of Brz99 from the case of a base field to the case of a commutative ground ring. In the special case $\varrho(a)=\sum a_{\psi} \otimes x^{\psi}$, for some group-like element $x \in C$, we get a complete generalization of [DM92, Theorem 1.5 ] (and Doi94, Theorem 2.5]).

With $A$ we denote a not necessarily commutative ring with $1_{A} \neq 0_{A}$ and with $\mathcal{M}_{A}$ (resp. ${ }_{A} \mathcal{M},{ }_{A} \mathcal{M}_{A}$ ) the category of unital right $A$-modules (resp. left $A$-modules, $A$-bimodules). For every right $A$-module $W$ we denote by $\operatorname{Gen}\left(W_{A}\right)$ (resp. $\sigma\left[W_{A}\right]$ ) the class of $W$-generated (resp. $W$-subgenerated) right $A$-modules. For the well developed theory of categories of type $\sigma[W]$ the reader is referred to [Wis88, Section 15].

An $A$-module $W$ is called locally projective (in the sense of B. Zimmermann-Huignes [Z-H76]), if for every diagram

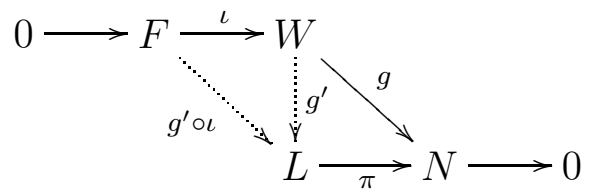

with exact rows and $F$ f.g.: for every $A$-linear map $g: W \longrightarrow N$, there exists an $A$-linear map $g^{\prime}: W \longrightarrow L$, such that the entstanding parallelogram is commutative. Note that every projective $A$-module is locally projective. By [Z-H76, Theorem 2.1] a left $A$-module $W$ is locally projective, iff for every right $A$-module $M$ the following map is injective

$$
\alpha_{M}^{W}: M \otimes_{A} W \longrightarrow \operatorname{Hom}_{-A}\left({ }^{*} W, M\right), m \otimes_{A} w \mapsto[f \mapsto m f(w)] .
$$

It's easy then to see that every locally projective $A$-module is flat and $A$-cogenerated.

Let $\mathcal{C}$ be an $A$-coring. We consider the canonical $A$-bimodule ${ }^{*} \mathcal{C}:=\operatorname{Hom}_{A-}(\mathcal{C}, A)$ as an $A$-ring with the canonical $A$-bimodule structure, multiplication $(f \cdot g)(c):=\sum g\left(c_{1} f\left(c_{2}\right)\right)$ and unity $\varepsilon_{\mathcal{C}}$. If ${ }_{A} \mathcal{C}$ is locally projective, then we have an isomorphism of categories $\mathcal{M}^{\mathcal{C}} \simeq$ $\sigma\left[\mathcal{C}_{*_{\mathcal{C}}}\right]$ (in particular $\mathcal{M}^{\mathcal{C}} \subseteq \mathcal{M}_{*_{\mathcal{C}}}$ is a full subcategory) and we have a left exact functor $\operatorname{Rat}^{\mathcal{C}}(-): \mathcal{M}^{*} \mathcal{C} \rightarrow \mathcal{M}^{\mathcal{C}}$ assigning to every right ${ }^{*} \mathcal{C}$-module its maximum $\mathcal{C}$-rational ${ }^{*} \mathcal{C}$ submodule, which turns to be a right $\mathcal{C}$-comodule. Moreover $\mathcal{M}^{\mathcal{C}}=\mathcal{M}^{*} \mathcal{C}$ iff ${ }_{A} \mathcal{C}$ is f.g. and projective. For more investigation of the $\mathcal{C}$-rational ${ }^{*} \mathcal{C}$-modules see Abu03.

After this paper was finished, it turned out that some results in this paper were discovered independently by S. Caenepeel, J. Vercruysse and S. Wang CVW04.

\section{Morita Contexts}

In this section we fix the following: $\mathcal{C}$ is an $A$-coring with group-like element $\mathbf{x}$ and $A$ is a right $\mathcal{C}$-comodule with structure map

$$
\varrho_{A}: A \longrightarrow A \otimes_{A} \mathcal{C} \simeq \mathcal{C}, a \mapsto \mathbf{x} a
$$

(e.g. [Brz02, Lemma 5.1]), hence $A \in \mathcal{M}_{* \mathcal{C}}$ with $a<g:=\sum a_{<0>} g\left(a_{<1>}\right)=g(\mathbf{x} a)$ for all $a \in A$ and $g \in{ }^{*} \mathcal{C}$. For $M \in \mathcal{M}^{*} \mathcal{C}$ put

$$
M^{\mathbf{x}}:=\left\{m \in M \mid m g=m g(\mathbf{x}) \text { for all } g \in{ }^{*} \mathcal{C}\right\} .
$$


In particular $A^{\mathbf{x}}:=\left\{a \in A \mid a\left\llcorner g=a g(\mathbf{x})\right.\right.$ for all $\left.g \in{ }^{*} \mathcal{C}\right\} \subset A$ is a subring. For $M \in \mathcal{M}^{\mathcal{C}}$ we set

$$
M^{c o \mathcal{C}}:=\left\{m \in M \mid \varrho(m)=m \otimes_{A} \mathbf{x}\right\} \subseteq M^{\mathbf{x}} .
$$

Obviously $B:=A^{c o \mathcal{C}}=\{b \in A \mid b \mathbf{x}=\mathbf{x} b\} \subseteq A^{\mathbf{x}}$ is a subring and $\varrho_{A}$ is $(B, A)$-bilinear. For $M \in \mathcal{M}^{\mathcal{C}}$ we have $M^{c o \mathcal{C}} \in \mathcal{M}_{B}$. Moreover we set

$$
Q:=\left\{q \in{ }^{*} \mathcal{C} \mid \sum c_{1} q\left(c_{2}\right)=q(c) \mathbf{x} \text { for all } c \in \mathcal{C}\right\} \subseteq\left({ }^{*} \mathcal{C}\right)^{\mathbf{x}}
$$

Lemma 1.1. 1. For every right ${ }^{*} \mathcal{C}$-module $M$ we have an isomorphism of right $B$ modules

$$
\omega_{M}: \operatorname{Hom}_{-* \mathcal{C}}(A, M) \longrightarrow M^{\mathbf{x}}, f \mapsto f\left(1_{A}\right)
$$

with inverse $m \mapsto[a \mapsto m a]$.

2. Let ${ }_{A} \mathcal{C}$ be locally projective. If $M \in \mathcal{M}^{\mathcal{C}}$, then $M^{\text {coC }}=M^{\mathbf{x}} \simeq \operatorname{Hom}_{-*} \mathcal{C}(A, M)=$ $\operatorname{Hom}^{\mathcal{C}}(A, M)$. Hence

$$
\Psi_{M}: M^{c o \mathcal{C}} \otimes_{B} A \longrightarrow M, m \otimes_{B} a \mapsto m a
$$

is surjective (resp. injective, bijective), iff

$$
\Psi_{M}^{\prime}: \operatorname{Hom}^{\mathcal{C}}(A, M) \otimes_{B} A \longrightarrow M, f \otimes_{B} a \mapsto f(a)
$$

is surjective (resp. injective, bijective).

3. We have $\operatorname{Hom}_{-{ }^{*} \mathcal{C}}\left(A,{ }^{*} \mathcal{C}\right) \simeq\left({ }^{*} \mathcal{C}\right)^{\mathbf{x}}$. If moreover ${ }_{A} \mathcal{C}$ is $A$-cogenerated (resp. locally projective and $\left.{ }^{\square} \mathcal{C}:=\operatorname{Rat}^{\mathcal{C}}\left({ }^{*} \mathcal{C}_{*} \mathcal{C}\right)\right)$, then $Q=\left({ }^{*} \mathcal{C}\right)^{\mathbf{x}}\left(\right.$ resp. $\left.Q=\left({ }^{\square} \mathcal{C}\right)^{\text {coC }}\right)$.

4. For every $M \in \mathcal{M}_{*_{\mathcal{C}}}$ (resp. $\left.M \in \mathcal{M}^{\mathcal{C}}\right)$ and all $m \in M, q \in Q$ we have $m q \in M^{\mathbf{x}}$ (resp. $m q \in M^{\text {coC }}$ ).

Proof. 1. Obvious.

2. Trivial.

3. Considering ${ }^{*} \mathcal{C}$ as a right ${ }^{*} \mathcal{C}$-module via right multiplication we get $\operatorname{Hom}_{-*}\left(A,{ }^{*} \mathcal{C}\right) \simeq$ $\left({ }^{*} \mathcal{C}\right)^{\mathbf{x}}$ by $(1)$. If $q \in\left({ }^{*} \mathcal{C}\right)^{\mathbf{x}}$, then we have for all $g \in{ }^{*} \mathcal{C}$ and $c \in \mathcal{C}$ :

$$
g\left(\sum c_{1} q\left(c_{2}\right)\right)=\sum g\left(c_{1} q\left(c_{2}\right)\right)=(q \cdot g)(c)=(q g(\mathbf{x}))(c)=q(c) g(\mathbf{x})=g(q(c) \mathbf{x})
$$

i.e. $\sum c_{1} q\left(c_{2}\right)-q(c) \mathbf{x} \in \operatorname{Re}(\mathcal{C}, A):=\bigcap\left\{\operatorname{Ke}(g) \mid g \in \operatorname{Hom}_{A-}(\mathcal{C}, A)\right\}$. If ${ }_{A} \mathcal{C}$ is $A$ cogenerated, then $\operatorname{Re}(\mathcal{C}, A)=0$, hence $Q=\left({ }^{*} \mathcal{C}\right)^{\mathbf{x}}$.

Assume ${ }_{A} \mathcal{C}$ to be locally projective. Then we have for all $q \in Q, g \in{ }^{*} \mathcal{C}$ and $c \in \mathcal{C}$ :

$$
(q \cdot g)(c)=\sum g\left(c_{1} q\left(c_{2}\right)\right)=g(q(c) \mathbf{x})=q(c) g(\mathbf{x})=(q g(\mathbf{x}))(c)
$$

hence $q \in \square_{\mathcal{C}}$, with $\varrho(q)=q \otimes_{A} \mathbf{x}$, i.e. $q \in\left(\square_{\mathcal{C}}\right)^{c o \mathcal{C}}$. On the other hand, if $q \in\left(\square_{\mathcal{C}}\right)^{\text {coC }}$, then for all $g \in{ }^{*} \mathcal{C}$ we have $q \cdot g=q g(\mathbf{x})$, i.e. $q \in\left({ }^{*} \mathcal{C}\right)^{\mathbf{x}}=Q$. 
4. Let $M \in \mathcal{M}^{*} \mathcal{C}$. Then we have for all $q \in Q, g \in{ }^{*} \mathcal{C}$ and $m \in M$ :

$$
(m q) g=m(q \cdot g)=m(q g(\mathbf{x}))=(m q) g(\mathbf{x}),
$$

i.e. $m q \in M^{\mathrm{x}}$. If $M \in \mathcal{M}^{\mathcal{C}}$, then we have for all $m \in M$ and $q \in Q$ :

$$
\begin{aligned}
\varrho_{M}(m q) & =\varrho_{M}\left(\sum m_{<0>} q\left(m_{<1>}\right)\right) \\
& =\sum m_{<0><0>} \otimes_{A} m_{<0><1>} q\left(m_{<1>}\right) \\
& =\sum m_{<0>} \otimes_{A} m_{<1>1} q\left(m_{<1>2}\right) \\
& =\sum m_{<0>} \otimes_{A} q\left(m_{<1>}\right) \mathbf{x} \\
& =\sum m_{<0>} q\left(m_{<1>}\right) \otimes_{A} \mathbf{x} \\
& =\sum m q \otimes_{A} \mathbf{x},
\end{aligned}
$$

i.e. $m q \in M^{c o C}$.

Lemma 1.2. 1. With the canonical actions $A$ is a $\left(B,{ }^{*} \mathcal{C}\right)$-bimodule.

2. $Q$ is a $\left({ }^{*} \mathcal{C}, B\right)$-bimodule.

Proof. 1. By assumption $A \in \mathcal{M}^{\mathcal{C}} \subseteq \mathcal{M}^{*} \mathcal{C}$. For all $b \in B, a \in A$ and $g \in{ }^{*} \mathcal{C}$ we have

$$
b(a<g)=b g(\mathbf{x} a)=g(b(\mathbf{x} a))=g(\mathbf{x}(b a))=(b a)<g .
$$

2. For all $a \in A, q \in Q$ and $c \in \mathcal{C}$ we have

$$
\sum c_{1}(a q)\left(c_{2}\right)=\sum c_{1} q\left(c_{2} a\right)=\sum(c a)_{1} q\left((c a)_{2}\right)=q(c a) \mathbf{x}=(a q)(c) \mathbf{x} .
$$

For all $q \in Q, b \in B$ and $c \in \mathcal{C}$ we have

$$
\sum c_{1}(q b)\left(c_{2}\right)=\sum c_{1} q\left(c_{2}\right) b=q(c) \mathbf{x} b=q(c) b \mathbf{x}=(q b)(c) \mathbf{x} .
$$

On the other hand we have for all $q \in Q, g \in{ }^{*} \mathcal{C}$ and $c \in \mathcal{C}$ :

$$
\begin{aligned}
& \sum c_{1}(g \cdot q)\left(c_{2}\right)=\sum c_{1} q\left(c_{21} g\left(c_{22}\right)\right)=\sum c_{11} q\left(c_{12} g\left(c_{2}\right)\right) \\
& =\sum c_{11}\left(g\left(c_{2}\right) q\right)\left(c_{12}\right)=\sum\left(g\left(c_{2}\right) q\right)\left(c_{1}\right) \mathbf{x} \\
& =\sum q\left(c_{1} g\left(c_{2}\right)\right) \mathbf{x}=(g \cdot q)(c) \mathbf{x} \text {. }
\end{aligned}
$$

Moreover we have for all $b \in B, q \in Q, g \in{ }^{*} \mathcal{C}$ and $c \in \mathcal{C}$ :

$$
\begin{aligned}
& ((g \cdot q) b)(c)=(g \cdot q)(c) b=\sum q\left(c_{1} g\left(c_{2}\right)\right) b \\
& =\sum(q b)\left(c_{1} g\left(c_{2}\right)\right)=(g \cdot q b)(c) \text {. }
\end{aligned}
$$

Theorem 1.3. Keep the notation above fixed.

1. $\left(A^{\mathrm{x}},{ }^{*} \mathcal{C}, A,\left({ }^{*} \mathcal{C}\right)^{\mathrm{x}}, \widetilde{F}, \widetilde{G}\right)$ is a Morita context derived form $A^{*} \mathcal{C}$, where

$$
\begin{aligned}
& \widetilde{F}: \quad\left({ }^{*} \mathcal{C}\right)^{\mathrm{x}} \otimes_{A^{\mathrm{x}}} A \quad \longrightarrow{ }^{*} \mathcal{C}, \quad q \otimes_{A^{\mathrm{x}}} a \mapsto q a, \\
& \widetilde{G}: A \otimes_{*_{\mathcal{C}}}\left({ }^{*} \mathcal{C}\right)^{\mathrm{x}} \longrightarrow A^{\mathrm{x}}, \quad a \otimes_{*_{\mathcal{C}}} q \quad \mapsto a<q .
\end{aligned}
$$


2. $\left(B,{ }^{*} \mathcal{C}, A, Q, F, G\right)$ is a Morita context, where

$$
\begin{aligned}
& F: Q \otimes_{B} A \longrightarrow{ }^{*} \mathcal{C}, \quad q \otimes_{B} a \mapsto q a, \\
& G: A \otimes_{*_{\mathcal{C}}} Q \quad \longrightarrow B, \quad a \otimes_{*_{\mathcal{C}}} q \quad \mapsto \quad a<q .
\end{aligned}
$$

If moreover ${ }_{A} \mathcal{C}$ is locally projective, then the two Morita contexts coincide.

Proof. 1. By Lemma 1.1 we have $\operatorname{End}\left(A_{{ }^{*} \mathcal{C}}\right) \simeq A^{\mathrm{x}},\left({ }^{*} \mathcal{C}\right)^{\mathrm{x}} \simeq \operatorname{Hom}_{-{ }^{*} \mathcal{C}}\left(A,{ }^{*} \mathcal{C}\right)$ and the result follows by [Fai81, Proposition 12.6].

2. By Lemma 1.2 $A$ is a $\left(B,{ }^{*} \mathcal{C}\right)$-bimodule and $Q$ is a $\left({ }^{*} \mathcal{C}, B\right)$-bimodule. For all $q \in Q, g \in$ ${ }^{*} \mathcal{C}, a \in A$ and $c \in \mathcal{C}$ we have

$$
F\left(g \cdot q \otimes_{B} a\right)(c)=\sum q\left(c_{2} g\left(c_{1}\right)\right) a=(g \cdot q a)(c)=\left(g \cdot F\left(q \otimes_{B} a\right)\right)(c)
$$

and

$$
\begin{array}{rlrl}
F\left(q \otimes_{B} a\llcorner g)(c)\right. & =q(c)(a\llcorner g) & & =q(c) g(\mathbf{x} a) \\
& =g(q(c) \mathbf{x} a) & & =\sum g\left(c_{1} q\left(c_{2}\right) a\right) \\
& =\sum g\left(c_{1}(q a)\left(c_{2}\right)\right) & =\left(F\left(q \otimes_{B} a\right) \cdot g\right)(c),
\end{array}
$$

hence $F$ is ${ }^{*} \mathcal{C}$-bilinear. Note that by Lemma $1.1 G$ is well defined and is obviously $B$-bilinear. Moreover we have for all $a, \widetilde{a} \in A$ and $q, \widetilde{q} \in Q$ the following associativity relations:

$$
\begin{array}{rlrl}
\left(F\left(q \otimes_{B} a\right) \cdot \widetilde{q}\right)(c) & =\sum \widetilde{q}\left(c_{1} q\left(c_{2}\right) a\right) & =\widetilde{q}(q(c) \mathbf{x} a) \\
& =q(c) \widetilde{q}(\mathbf{x} a) & & =\left(q G\left(a \otimes_{\mathcal{C}} \widetilde{q}\right)\right)(c), \\
G\left(a \otimes_{*_{\mathcal{C}}} q\right) \widetilde{a} & =q(\mathbf{x} a) \widetilde{a} & & =(q \widetilde{a})(\mathbf{x} a) \\
& =F\left(q \otimes_{B} \widetilde{a}\right)(\mathbf{x} a) & =a \leftarrow F\left(q \otimes_{B} \widetilde{a}\right) .
\end{array}
$$

If ${ }_{A} \mathcal{C}$ is locally projective, then $A^{\mathrm{x}}=A^{c o \mathcal{C}},\left({ }^{*} \mathcal{C}\right)^{\mathrm{x}}=Q$ by Lemma 1.1 and the two contexts coincide.

1.4. Brz02, Definition 5.3] An $A$-coring $\mathcal{C}$ is said to be Galois, if there exists an $A$-coring isomorphism $\chi: A \otimes_{B} A \longrightarrow \mathcal{C}$ such that $\chi\left(1_{A} \otimes_{B} 1_{A}\right)=\mathrm{x}$. Recall that $A \otimes_{B} A$ is an $A$-coring with the canonical $A$-bimodule structure, comultiplication

$$
\Delta: A \otimes_{B} A \longrightarrow\left(A \otimes_{B} A\right) \otimes_{A}\left(A \otimes_{B} A\right), \widetilde{a} \otimes_{B} a \mapsto\left(\widetilde{a} \otimes_{B} 1_{A}\right) \otimes_{A}\left(1_{A} \otimes_{B} a\right)
$$

and counity $\varepsilon_{A \otimes_{B} A}: A \otimes_{B} A \longrightarrow A, \widetilde{a} \otimes_{B} a \mapsto \widetilde{a} a$.

1.5. Consider the functors

$$
(-)^{c o \mathcal{C}}: \mathcal{M}^{\mathcal{C}} \longrightarrow \mathcal{M}_{B} \text { and }-\otimes_{B} A: \mathcal{M}_{B} \longrightarrow \mathcal{M}^{\mathcal{C}} .
$$

By [Brz02, Proposition 5.2] $\left(-\otimes_{B} A,(-)^{c o \mathcal{C}}\right)$ is an adjoint pair of covariant functors, where the adjunctions are given by

$$
\Phi_{N}: N \longrightarrow\left(N \otimes_{B} A\right)^{c c \mathcal{C}}, n \mapsto n \otimes_{B} 1_{A}
$$


and

$$
\Psi_{M}: M^{c o C} \otimes_{B} A \longrightarrow M, m \otimes_{B} a \mapsto m a .
$$

If $\Psi_{M}$ is an isomorphism for all $M \in \mathcal{M}^{\mathcal{C}}$, then we say $\mathcal{M}^{\mathcal{C}}$ satisfies the weak structure theorem. If in addition $\Phi_{N}$ is an isomorphism for all $N \in \mathcal{M}_{B}$, then we say $\mathcal{M}^{\mathcal{C}}$ satisfies the strong structure theorem (in this case $(-)^{c o C}$ and $-\otimes_{B} A$ give an equivalence of categories $\mathcal{M}^{\mathcal{C}} \simeq \mathcal{M}_{B}$ ).

1.6. Let $W \in \mathcal{M}_{A}$ and consider the canonical right $\mathcal{C}$-comodule $W \otimes_{A} \mathcal{C}$. Then $W \simeq$ $\left(W \otimes_{A} \mathcal{C}\right)^{c o \mathcal{C}}$ via $w \mapsto w \otimes_{A} \mathbf{x}$ with inverse $w \otimes_{A} c \mapsto w \varepsilon_{\mathcal{C}}(c)$ and we define

$$
\beta_{W}:=\Psi_{W \otimes_{A} \mathcal{C}}: W \otimes_{B} A \longrightarrow W \otimes_{A} \mathcal{C}, w \otimes_{B} a \mapsto w \otimes_{A} \mathbf{x} a
$$

In particular we have for $W=A$ the morphism of $A$-corings

$$
\beta:=\Psi_{A \otimes_{A} \mathcal{C}}: A \otimes_{B} A \longrightarrow A \otimes_{A} \mathcal{C} \simeq \mathcal{C}, \tilde{a} \otimes_{B} a \mapsto \widetilde{a} \mathbf{x} a
$$

If $\beta$ is bijective, then $\mathcal{C}$ is a Galois $A$-coring and we call the ring extension $A / B \mathcal{C}$-Galois.

Theorem 1.7. For the Morita context $\left(B,{ }^{*} \mathcal{C}, A, Q, F, G\right)$ the following statements are equivalent:

1. $G: A \otimes *_{\mathcal{C}} Q \longrightarrow B$ is surjective (bijective and $B=A^{\mathbf{x}}$ );

2. there exists $\widehat{q} \in Q$, such that $\widehat{q}(\mathbf{x})=1_{A}$;

3. for every right ${ }^{*} \mathcal{C}$-module $M$ we have a $B$-module isomorphism $M \otimes_{*_{\mathcal{C}}} Q \simeq M^{\mathbf{x}}$.

4. for every right $\mathcal{C}$-comodule $M$ we have $M \otimes_{*_{\mathcal{C}}} Q \simeq M^{\mathrm{coC}}$ as $B$-modules.

If moreover ${ }_{A} \mathcal{C}$ is locally projective, then (1)-(4) are moreover equivalent to:

5. $A_{* \mathcal{C}}$ is (f.g.) projective.

Proof. $(1) \Rightarrow(2)$. Assume $G$ to be surjective. Then there exist $a_{1}, \ldots, a_{k}$ and $q_{1}, \ldots, q_{k} \in Q$, such that $G\left(\sum_{i=1}^{k} a_{i} \otimes{ }^{*} \mathcal{C} q_{i}\right)=1_{A}$. Set $\widehat{q}:=\sum_{i=1}^{k} a_{i} q_{i} \in Q$. Then we have

$$
\widehat{q}(\mathbf{x})=\left(\sum_{i=1}^{k} a_{i} q_{i}\right)(\mathbf{x})=\sum_{i=1}^{k} q_{i}\left(\mathbf{x} a_{i}\right)=\sum_{i=1}^{k}\left(a_{i} \leftarrow q_{i}\right)=G\left(\sum_{i=1}^{k} a_{i} \otimes_{*_{\mathcal{C}}} q_{i}\right)=1_{A}
$$

$(2) \Rightarrow(3)$. Consider the $B$-module morphism

$$
\xi_{M}: M \otimes *_{\mathcal{C}} Q \longrightarrow M^{\mathrm{x}}, m \otimes *_{\mathcal{C}} q \mapsto m q
$$

Let $\widehat{q} \in Q$ with $\widehat{q}(\mathbf{x})=1_{A}$ and define $\widetilde{\xi}_{M}: M^{\mathbf{x}} \longrightarrow M \otimes_{*_{\mathcal{C}}} Q, m \mapsto m \otimes_{*_{\mathcal{C}}} \widehat{q}$. For every $n \in M^{\mathrm{x}}$ we have

$$
\left(\xi_{M} \circ \widetilde{\xi}_{M}\right)(n)=\xi_{M}\left(n \otimes_{* \mathcal{C}} \widehat{q}\right)=n\llcorner\widehat{q}=n \widehat{q}(\mathbf{x})=n .
$$


On the other hand we have for all $m \in M$ and $q \in Q$ :

$$
\begin{aligned}
\left(\widetilde{\xi}_{M} \circ \xi_{M}\right)\left(m \otimes *_{\mathcal{C}} q\right) & =\widetilde{\xi}_{M}\left(m\llcorner q)=m<q \otimes_{*_{\mathcal{C}}} \widehat{q}=m \otimes_{*_{\mathcal{C}}} q \cdot \widehat{q}\right. \\
& =m \otimes *_{\mathcal{C}} q \widehat{q}(\mathbf{x})=m \otimes *_{\mathcal{C}} q,
\end{aligned}
$$

i.e. $\xi_{M}$ is bijective with inverse $\widetilde{\xi}_{M}$.

$(3) \Rightarrow(4)$. Let $M \in \mathcal{M}^{\mathcal{C}}$. By Lemma 1.1 we have $\xi_{M}\left(M \otimes_{*_{\mathcal{C}}} Q\right) \subseteq M^{c o \mathcal{C}} \subseteq M^{\mathrm{x}}$. By assumption $\xi_{M}: A \otimes_{*_{\mathcal{C}}} Q \longrightarrow M^{\mathrm{x}}$ is bijective. Hence $M^{\mathbf{x}}=M^{c o \mathcal{C}}$ and $M \otimes_{*_{\mathcal{C}}} Q \stackrel{\xi_{M}}{\simeq} M^{c o \mathcal{C}}$. $(4) \Rightarrow(1)$ We are done since $G=\xi_{A}$.

Assume ${ }_{A} \mathcal{C}$ to be locally projective.

Then $B \simeq \operatorname{End}\left(A_{*^{*} \mathcal{C}}\right), Q \simeq \operatorname{Hom}_{-{ }^{*} \mathcal{C}}\left(A,{ }^{*} \mathcal{C}\right)$ and we get $(1) \Longleftrightarrow(5)$ by [Fai81, Corollary $12.8]$.

Corollary 1.8. For the Morita context $\left(B,{ }^{*} \mathcal{C}, A, Q, F, G\right)$ assume there exists $\widehat{q} \in Q$ with $\widehat{q}(\mathbf{x})=1_{A}$ (equivalently $G: Q \otimes_{B} A \longrightarrow{ }^{*} \mathcal{C}$ is surjective). Then:

1. For every $N \in \mathcal{M}_{B}, \Phi_{N}$ is an isomorphism.

2. $B$ is a left $B$-direct summand of $A$.

Proof. 1. Let $N \in \mathcal{M}_{B}$. Then we have by Theorem 1.7 the isomorphisms $G: A \otimes_{* \mathcal{C}}$ $Q \longrightarrow B$ and $\xi_{N \otimes_{B} A}:\left(N \otimes_{B} A\right) \otimes_{*_{\mathcal{C}}} Q \longrightarrow\left(N \otimes_{B} A\right)^{c o \mathcal{C}}$. Moreover $\Phi_{N}$ is given by the canonical isomorphisms

$$
N \simeq N \otimes_{B} B \simeq N \otimes_{B}\left(A \otimes_{*_{\mathcal{C}}} Q\right) \simeq\left(N \otimes_{B} A\right) \otimes_{*_{\mathcal{C}}} Q \simeq\left(N \otimes_{B} A\right)^{c o \mathcal{C}}
$$

2. The map $\operatorname{tr}_{A}: A \longrightarrow B, a \mapsto a<\widehat{q}$ is left $B$-linear with $\operatorname{tr}_{A}(b)=b$ for all $b \in B$.

Corollary 1.9. For the Morita context $\left(B,{ }^{*} \mathcal{C}, A, Q, F, G\right)$ assume there exists $\widehat{q} \in Q$ with $\widehat{q}(\mathbf{x})=1_{A}$ (equivalently $G: Q \otimes_{B} A \longrightarrow B$ is surjective). Then:

1. ${ }_{B} A$ and $Q_{B}$ are generators.

2. $A_{*_{\mathcal{C}}}$ and ${ }^{*} \mathcal{C} Q$ are f.g. and projective.

3. $F: Q \otimes_{B} A \longrightarrow{ }^{*} \mathcal{C}$ induces bimodule isomorphisms

$$
A \simeq \operatorname{Hom}_{* \mathcal{C}-}\left(Q,{ }^{*} \mathcal{C}\right) \text { and } Q \simeq \operatorname{Hom}_{-*} \mathcal{C}\left(A,{ }^{*} \mathcal{C}\right)
$$

4. The bimodule structures above induce ring isomorphisms

$$
B \simeq \operatorname{End}\left(A *_{\mathcal{C}}\right) \text { and } B \simeq \operatorname{End}\left({ }^{*} \mathcal{C} Q\right)^{o p} .
$$

Proof. The result follows by standard argument of Morita Theory (e.g. Fai81, Proposition $12.7])$.

Proposition 1.10. Consider the Morita context $\left(B,{ }^{*} \mathcal{C}, A, Q, F, G\right)$ and assume that $F$ : $Q \otimes *_{\mathcal{C}} A \longrightarrow{ }^{*} \mathcal{C}$ is surjective. Then: 
1. $A^{*} \mathcal{C}$ is a generator, $Q \simeq \operatorname{Hom}_{B-}(A, B)$ as bimodules and ${ }^{*} \mathcal{C} \simeq \operatorname{End}\left(Q_{B}\right)$.

2. $\mathcal{M}^{\mathcal{C}}$ satisfies the weak structure theorem (in particular $A / B$ is $\mathcal{C}$-Galois).

Proof. 1. The result follows by standard argument of Morita Theory (e.g. Fai81, Proposition 12.7]).

2. By assumption $\varepsilon_{\mathcal{C}}=F\left(\sum_{i=1}^{k} q_{i} \otimes_{B} a_{i}\right)$ for some $\left\{\left(q_{i}, a_{i}\right)\right\}_{i=1}^{k} \subseteq Q \times A$. In this case $\Psi_{M}: M^{c o \mathcal{C}} \otimes_{B} A \longrightarrow M$ is bijective with inverse $\widetilde{\Psi}_{M}: M \longrightarrow M^{c o \mathcal{C}} \otimes_{B} A, m \mapsto$ $\sum_{i=1}^{k} m q_{i} \otimes_{B} a_{i}$. In fact, we have for all $m \in M, n \in M^{c o \mathcal{C}}$ and $a \in A$ :

$$
\begin{array}{rlrl}
\left(\Psi_{M} \circ \widetilde{\Psi}_{M}\right)(m) & =\sum_{i=1}^{k}\left(m q_{i}\right) a_{i} & =\sum_{i=1}^{k}\left(m_{<0>} q_{i}\left(m_{<1>}\right) a_{i}\right. \\
& =\sum_{i=1}^{k} m_{<0>}\left(q_{i} a_{i}\right)\left(m_{<1>}\right)=\sum_{i=1}^{k} m_{<0>} \varepsilon_{\mathcal{C}}\left(m_{<1>}\right) \\
& =m
\end{array}
$$

and

$$
\begin{aligned}
& \left(\widetilde{\Psi}_{M} \circ \Psi_{M}\right)\left(n \otimes_{B} a\right)=\sum_{i=1}^{k}(n a) q_{i} \otimes_{B} a_{i}=\sum_{i=1}^{k} n q_{i}(\mathbf{x} a) \otimes_{B} a_{i} \\
& =\sum_{i=1}^{k} n \otimes_{B} q_{i}(\mathbf{x} a) a_{i}=\sum_{i=1}^{k} n \otimes_{B}\left(q_{i} a_{i}\right)(\mathbf{x} a) \\
& =n \otimes_{B} \varepsilon_{\mathcal{C}}(\mathbf{x} a)=n \otimes_{B} a \text {. }
\end{aligned}
$$

Theorem 1.11. For the Morita context $\left(B,{ }^{*} \mathcal{C}, A, Q, F, G\right)$ the following are equivalent:

1. $F: Q \otimes_{B} A \longrightarrow{ }^{*} \mathcal{C}$ is surjective (bijective);

2. (a) $Q_{B}$ is f.g. and projective;

(b) $\Omega: A \longrightarrow \operatorname{Hom}_{-B}(Q, B), a \mapsto[q \mapsto a<q]$ is a bimodule isomorphism;

(c) ${ }^{*} \mathcal{C} Q$ is faithful.

If ${ }_{A} \mathcal{C}$ is $A$-cogenerated, then (1) \& (2) are moreover equivalent to:

3. (a) ${ }_{B} A$ is f.g. and projective;

(b) $\Lambda:{ }^{*} \mathcal{C} \longrightarrow \operatorname{End}\left({ }_{B} A\right)^{o p}, g \mapsto[a \mapsto a<g]$ is a ring isomorphism.

4. $A{ }^{*} \mathrm{C}$ is a generator.

If moreover ${ }_{A} \mathcal{C}$ is f.g. and projective, then (1)-(4) are equivalent to:

5. $\mathcal{M}^{\mathcal{C}}$ satisfies the weak structure theorem. 
Proof. The implications (1) $\Rightarrow(2),(3),(4)$ follow without any finiteness conditions on $\mathcal{C}$ by standard argument of Morita Theory (e.g. [Fai81, Proposition 12.7]). Note that ${ }^{*} \mathcal{C} Q$ is faithful by the embedding ${ }^{*} \mathcal{C} \hookrightarrow \operatorname{End}\left(Q_{B}\right)$ (see Proposition $1.10(1)$ ).

$(2) \Rightarrow(1)$. Let $\left\{\left(q_{i}, p_{i}\right)\right\}_{i=1}^{k} \subset Q \times \operatorname{Hom}_{-B}(Q, B)$ be a dual basis for $Q_{B}$. By (b) there exist $a_{1}, \ldots, a_{k} \in A$, such that $\Omega\left(a_{i}\right)=q_{i}$ for $i=1, \ldots, k$. For every $q \in Q$ we have then

$\left(\sum_{i=1}^{k} q_{i} a_{i}\right) \cdot q=\sum_{i=1}^{k} q_{i}\left(a_{i}<q\right)=\sum_{i=1}^{k} q_{i} p_{i}(q)=q$, hence $\sum_{i=1}^{k} q_{i} a_{i}=\varepsilon_{\mathcal{C}}$ by $(\mathrm{c})$ and the ${ }^{*} \mathcal{C}$-bilinear morphism $F: Q \otimes_{*_{\mathcal{C}}} A \longrightarrow{ }^{*} \mathcal{C}$ is surjective.

Assume ${ }_{A} \mathcal{C}$ to be $A$-cogenerated.

(3) $\Rightarrow(1)$. Let $\left\{\left(a_{i}, p_{i}\right)\right\}_{i=1}^{k} \subset A \times \operatorname{Hom}_{B-}(A, B)$ be a dual basis of ${ }_{B} A$. By (b), there exist $g_{1}, \ldots, g_{k} \in{ }^{*} \mathcal{C}$, such that $\Lambda\left(g_{i}\right)=p_{i}$ for $i=1, \ldots, k$. Claim: $g_{1}, \ldots, g_{k} \in Q$. For all $f \in$ ${ }^{*} \mathcal{C}$ and $i=1, \ldots, k$ we have

$$
\begin{aligned}
\Lambda\left(g_{i} \cdot f\right)(a) & \left.=a<\left(g_{i} \cdot f\right)=\left(a<g_{i}\right)<f\right) \\
& =p_{i}(a) \leftarrow f=f\left(\mathbf{x} p_{i}(a)\right) \\
& =f\left(p_{i}(a) \mathbf{x}\right)=p_{i}(a) f(\mathbf{x}) \\
& =\left(p_{i} f(\mathbf{x})\right)(a)=\Lambda\left(g_{i} f(\mathbf{x})\right)(a)
\end{aligned}
$$

hence $g_{i} \cdot f=g_{i} f(\mathbf{x})$, i.e. $g_{i} \in\left({ }^{*} \mathcal{C}\right)^{\mathbf{x}}=Q$ (by Lemma 1.1 (2)). Moreover for every $a \in A$ we have: $\Lambda\left(\sum_{i=1}^{k} g_{i} a_{i}\right)(a)=\sum_{i=1}^{k} a<g_{i} a_{i}=\sum_{i=1}^{k} p_{i}(a) a_{i}=a$, i.e. $\sum_{i=1}^{k} g_{i} a_{i}=\varepsilon_{\mathcal{C}}$ and the ${ }^{*} \mathcal{C}$-bilinear morphism $F$ is surjective.

$(4) \Rightarrow(1)$. Since $Q \simeq \operatorname{Hom}_{-}{ }^{*} \mathcal{C}\left(A,{ }^{*} \mathcal{C}\right)$, we have $\operatorname{Im}(F)=\operatorname{tr}\left(A,{ }^{*} \mathcal{C}\right):=\sum\{\operatorname{Im}(h): h \in$ $\left.\operatorname{Hom}_{-}{ }^{*} \mathcal{C}\left(A,{ }^{*} \mathcal{C}\right)\right\}$, hence $\operatorname{Im}(F)={ }^{*} \mathcal{C}$ iff $A_{*} \mathcal{C}$ is a generator (e.g. [Wis88, Page 154]).

Assume ${ }_{A} \mathcal{C}$ to be f.g. and projective.

$(1) \Rightarrow(5)$ follows without any finiteness conditions on $\mathcal{C}$ by Proposition 1.10 (2).

(5) $\Rightarrow(1)$. Since ${ }_{A} \mathcal{C}$ is f.g. and projective, we have $\mathcal{M}^{\mathcal{C}} \simeq \mathcal{M}_{*_{\mathcal{C}}}$ (e.g. Brz02, Lemma 4.3]), hence ${ }^{*} \mathcal{C} \in \mathcal{M}^{\mathcal{C}}, Q=\left({ }^{*} \mathcal{C}\right)^{\text {coC }}$ and $F=\Psi_{*}$.

\section{Galois Extensions and Equivalences}

The notation of the first section remains fixed. For every $M \in \mathcal{M}^{\mathcal{C}}$ we have the $\mathcal{C}$-colinear morphism

$$
\Psi_{M}^{\prime}: \operatorname{Hom}^{\mathcal{C}}(A, M) \otimes_{B} A \longrightarrow M, f \otimes_{B} a \mapsto f(a) .
$$

In this section we characterize $A$ being a generator (resp. a progenerator) in $\mathcal{M}^{\mathcal{C}}$ under the assumption that ${ }_{A} \mathcal{C}$ is locally projective. Our approach is similar to that of [MZ97] and our results generalize those obtained there for the special case of the category of Doi-Koppinen modules $\mathcal{M}(H)_{A}^{C}$.

Lemma 2.1. Assume ${ }_{A} \mathcal{C}$ to be locally projective. If ${ }_{B} A$ is flat and $A / B$ is $\mathcal{C}$-Galois, then:

1. $A$ is a subgenerator in $\mathcal{M}^{\mathcal{C}}$, i.e. $\sigma\left[A_{*_{\mathcal{C}}}\right]=\sigma\left[\mathcal{C}_{*} \mathcal{C}\right.$.

2. for each $M \in \mathcal{M}^{\mathcal{C}}, \Psi_{M}^{\prime}$ is injective.

3. for every A-generated $M \in \mathcal{M}^{\mathcal{C}}, \Psi_{M}^{\prime}$ is an isomorphism. 
Proof. Assume ${ }_{A} \mathcal{C}$ to be locally projective.

1. Since $A / B$ is $\mathcal{C}$-Galois, $\beta^{\prime}:=\Psi_{\mathcal{C}}^{\prime}$ is an isomorphism, hence $\mathcal{C}$ is $A$-generated. Consequently $\sigma\left[A_{*_{\mathcal{C}}}\right] \subseteq \sigma\left[\mathcal{C}_{*_{\mathcal{C}}}\right] \subseteq \sigma\left[A_{*_{\mathcal{C}}}\right]$, i.e. $\sigma\left[A_{*_{\mathcal{C}}}\right]=\sigma\left[\mathcal{C}_{*_{\mathcal{C}}}\right]$.

2. With slight modifications, the proof of [MZ97, Lemma 3.22] applies.

3. If $M \in \mathcal{M}^{\mathcal{C}}$ is $A$-generated, then $\Psi_{M}^{\prime}$ is surjective, hence bijective by (2).

The following result is a generalization of [Brz99, Proposition 3.13] (which in turn generalizes [DT89, Theorem 2.11]):

Proposition 2.2. Assume $A / B$ to be $\mathcal{C}$-Galois.

1. If ${ }_{B} A$ is flat, then $\mathcal{M}^{\mathcal{C}}$ satisfies the weak structure theorem.

2. Assume there exists $\widehat{q} \in Q$, such that $\widehat{q}(\mathbf{x})=1_{A}$. If ${ }_{B} A$ is flat, or for all $b \in B$ and $c \in \mathcal{C}$ we have $\widehat{q}(c b)=q(c) b$, then $\mathcal{M}^{\mathcal{C}}$ satisfies the strong structure theorem.

Proof. 1. The proof is the first part of the proof of [Brz02, Theorem 5.6].

2. By assumption and Corollary 1.8, $\Phi_{N}$ is an isomorphism for all $N \in \mathcal{M}_{B}$. If ${ }_{B} A$ is flat, then $\mathcal{M}^{\mathcal{C}}$ satisfies the weak structure theorem by (1). On the other hand, if for all $b \in B$ and $c \in \mathcal{C}$ we have $\widehat{q}(c b)=q(c) b$, then an analog argument to that in the proof of [Brz99, Proposition 3.13] shows that $\mathcal{M}^{\mathcal{C}}$ satisfies the weak structure theorem.

Theorem 2.3. Assume ${ }_{A} \mathcal{C}$ to be locally projective. Then the following are equivalent:

1. $\mathcal{M}^{\mathcal{C}}$ satisfies the weak structure theorem;

2. ${ }_{B} A$ is flat and $A / B$ is $\mathcal{C}$-Galois;

3. ${ }_{B} A$ is flat and $\beta^{\prime}:=\Psi_{\mathcal{C}}^{\prime}$ is an isomorphism;

4. ${ }_{B} A$ is flat and for every $A$-generated $M \in \mathcal{M}^{\mathcal{C}}, \Psi_{M}^{\prime}$ is bijective;

5. for every $M \in \mathcal{M}^{\mathcal{C}}=\sigma\left[\mathcal{C}_{* \mathcal{C}}\right]$, the $\mathcal{C}$-colinear morphism $\Psi_{M}^{\prime}$ is bijective;

6. $\sigma\left[\mathcal{C}_{* \mathcal{C}}\right]=\operatorname{Gen}\left(A_{*_{\mathcal{C}}}\right)$;

7. ${ }_{B} A$ is flat, $\sigma\left[\mathcal{C}_{{ }^{*} \mathcal{C}}\right]=\sigma\left[A_{*_{\mathcal{C}}}\right]$ and $\operatorname{Hom}_{-{ }^{*} \mathcal{C}}(A,-): \operatorname{Gen}\left(A_{*_{\mathcal{C}}}\right) \longrightarrow \mathcal{M}_{B}$ is full faithful;

8. $\operatorname{Hom}^{\mathcal{C}}(A,-): \mathcal{M}^{\mathcal{C}} \longrightarrow \mathcal{M}_{B}$ is faithful;

9. $A$ is a generator in $\mathcal{M}^{\mathcal{C}}$.

Proof. $(1) \Longleftrightarrow(5) \&(2) \Longleftrightarrow(3)$ follow by Lemma 1.1 The equivalences $(4) \Longleftrightarrow(5) \Longleftrightarrow$ $(6) \Longleftrightarrow(7)$ follow by [MZ97, Theorem 2.3]. The equivalence $(8) \Longleftrightarrow(9)$ is evident for any category, and moreover $(6) \Longleftrightarrow(9)$ by the fact that $\operatorname{Gen}\left(A_{*_{\mathcal{C}}}\right) \subseteq \sigma\left[A_{{ }^{*} \mathcal{C}}\right] \subseteq \sigma\left[\mathcal{C}_{{ } \mathcal{C}}\right]=\mathcal{M}^{\mathcal{C}}$. By Lemma 2.1 we have $(3) \Rightarrow(4)$. Now assuming (1) we conclude that $A / B$ is $\mathcal{C}$-Galois and that ${ }_{B} A$ is flat (since $\left.(1) \Longleftrightarrow(5) \Longleftrightarrow(7)\right)$, hence $(1) \Rightarrow(2)$ follows and we are done. 
Definition 2.4. ([MZ97, Definition 2.4]) A left module $P$ over a ring $\mathcal{S}$ is called a weak generator, if for any right $\mathcal{S}$-module $Y, Y \otimes_{\mathcal{S}} P=0$ implies $Y=0$. A right module $P$ over a ring $\mathcal{R}$ is called quasiprogenerator (resp. progenerator), if $P_{\mathcal{R}}$ is f.g. quasiprojective and generates each of its submodules (resp. $P_{\mathcal{R}}$ is f.g., projective and a generator). $P_{\mathcal{R}}$ is called faithful (resp. balanced), if the canonical morphism $\mathcal{R} \longrightarrow \operatorname{End}\left(\operatorname{End}\left(P_{\mathcal{R}}\right) P\right)^{o p}$ is injective (resp. surjective).

Theorem 2.5. Assume ${ }_{A} \mathcal{C}$ to be flat. Then the following are equivalent:

1. $\mathcal{M}^{\mathcal{C}}$ satisfies the strong structure theorem;

2. ${ }_{B} A$ is faithfully flat and $A / B$ is $\mathcal{C}$-Galois.

If moreover ${ }_{A} \mathcal{C}$ is locally projective, then (1) \& (2) are moreover equivalent to:

3. ${ }_{B} A$ is faithfully flat and $\beta^{\prime}:=\Psi_{\mathcal{C}}^{\prime}$ is bijective;

4. ${ }_{B} A$ is faithfully flat and for every $M \in \sigma\left[A_{*}\right], \Psi_{M}^{\prime}$ is bijective;

5. $A_{*_{\mathcal{C}}}$ is quasiprojective and generates each of its submodules, ${ }_{B} A$ is a weak generator and $\sigma\left[\mathcal{C}_{* \mathcal{C}}\right]=\sigma\left[A_{*} \mathcal{C}\right.$;

6. $A_{*_{\mathcal{C}}}$ is a quasiprogenerator and $\sigma\left[\mathcal{C}_{*}\right]=\sigma\left[A_{*} \mathcal{C}\right]$;

7. ${ }_{B} A$ is a weak generator, $\Psi_{M}^{\prime}$ is an isomorphism for every $M \in \operatorname{Gen}\left(A_{*_{\mathcal{C}}}\right)$ and $\sigma\left[A_{*_{\mathcal{C}}}\right]=$ $\sigma\left[\mathcal{C}_{* \mathcal{C}}\right]$

8. $\operatorname{Hom}^{\mathcal{C}}(A,-): \mathcal{M}^{\mathcal{C}} \longrightarrow \mathcal{M}_{B}$ is an equivalence;

9. $A$ is a progenerator in $\mathcal{M}^{\mathcal{C}}$.

Proof. (1) $\Longleftrightarrow(2)$ is $\left[\mathrm{Brz02}\right.$, Theorem 5.6]. Assume ${ }_{A} \mathcal{C}$ to be locally projective. Then $(2) \Longleftrightarrow(3)$ follows by Lemma 1.1 and we get $(1) \Longleftrightarrow(8) \Longleftrightarrow(9)$ by characterizations of progenerators in categories of type $\sigma[M]$ (see Wis88, 18.5, 46.2]). Moreover (4) $(5) \Longleftrightarrow(6) \Longleftrightarrow(7)$ follow from [MZ97, Theorem 2.5]. Obviously (3) $\Rightarrow$ (4) (note that $(3) \Longleftrightarrow(2) \Longleftrightarrow(1))$. Assume now (4). Then ${ }_{B} A$ is faithfully flat and moreover $\Psi_{\mathcal{C}}^{\prime}$ is bijective, since $\mathcal{C} \in \sigma\left[A_{*} \mathcal{C}\right]$ by (6), i.e. (4) $\Rightarrow$ (3) and the proof is complete.

Remark 2.6. Assume ${ }_{A} \mathcal{C}$ to be locally projective. Then $\operatorname{Im}(F) \subseteq \square_{\mathcal{C}}$. In fact we have for all $q \in Q, a \in A, g \in{ }^{*} \mathcal{C}$ and $c \in C$ :

$$
((q a) \cdot g)(c)=\sum g\left(c_{1} q\left(c_{2}\right) a\right)=g(q(c) \mathbf{x} a)=q(c) g(\mathbf{x} a)=(q g(\mathbf{x} a)(c),
$$

hence $q a \in \square \mathcal{C}$, with $\varrho(q a)=q \otimes_{A} \mathbf{x} a$.

Proposition 2.7. Assume ${ }_{A} \mathcal{C}$ to be locally projective and that there exists $\widehat{q} \in Q$ with $\widehat{q}(\mathbf{x})=1_{A}$ (equivalently $G: A \otimes_{*_{\mathcal{C}}} Q \longrightarrow B$ is surjective). Then $\mathcal{M}^{\mathcal{C}}$ satisfies the strong equivalence theorem, iff $\operatorname{Im}(F)={ }^{\mathcal{C}}$ and the following map is surjective for every $M \in \mathcal{M}^{\mathcal{C}}$

$$
\varpi_{M}: M \otimes_{*_{\mathcal{C}}} \square_{\mathcal{C}} \longrightarrow M, m \otimes_{*_{\mathcal{C}}} f \mapsto m f .
$$

In this case $Q \otimes_{B} A \stackrel{F}{\simeq} \square_{\mathcal{C}}$ and $M \otimes_{*_{\mathcal{C}}} \square_{\mathcal{C}} \stackrel{\varpi_{M}}{\simeq} M$ for every $M \in \mathcal{M}^{\mathcal{C}}$. 
Proof. Consider for every $M \in \mathcal{M}^{\mathcal{C}}$ the commutative diagram

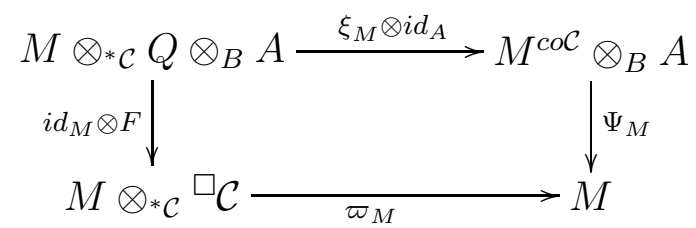

Assume $\operatorname{Im}(F)=\square_{\mathcal{C}}$ and $\varpi_{M}$ to be surjective for every $M \in \mathcal{M}^{\mathcal{C}}$. Then $\Psi_{M}$ is obviously surjective. Let $K=\operatorname{Ke}\left(\Psi_{M}\right)$. Since $\Psi_{M}$ is a morphism in $\mathcal{M}^{\mathcal{C}} \simeq \sigma\left[\mathcal{C}_{*_{\mathcal{C}}}\right]$ we have $K \in \mathcal{M}^{\mathcal{C}}$, hence $\Psi_{K}: K^{c o \mathcal{C}} \otimes_{B} A \longrightarrow K$ is surjective. By Theorem 1.7 we have $K \otimes_{*_{\mathcal{C}}} Q \stackrel{\xi_{K}}{\simeq} K^{c o \mathcal{C}}$ and $A \otimes *_{\mathcal{C}} Q \stackrel{\xi_{A}}{\simeq} B$, hence

$$
K^{c o \mathcal{C}} \simeq K \otimes_{*_{\mathcal{C}}} Q=\operatorname{Ke}\left(\Psi_{M}\right) \otimes_{*_{\mathcal{C}}} Q=\operatorname{Ke}\left(\Psi_{M} \otimes_{*_{\mathcal{C}}} i d_{Q}\right)=\operatorname{Ke}\left(i d_{M^{c o \mathcal{C}}}\right)=0,
$$

i.e. $\Psi_{M}$ is bijective. By corollary[1.8 $\Phi_{N}$ is bijective for every $N \in \mathcal{M}_{B}$. Consequently $\mathcal{M}^{\mathcal{C}}$ satisfies the strong structure theorem.

On the other hand, assume that $\mathcal{M}^{\mathcal{C}}$ satisfies the strong structure theorem. Note that $F$ is the adjunction of $\Psi_{\square_{\mathcal{C}}}$, hence $Q \otimes_{B} A \stackrel{F}{\simeq} \square_{\mathcal{C}}$ and consequently $\varpi_{M}$ is also bijective for every $M \in \mathcal{M}^{\mathcal{C}}$ by the commutativity of the above diagram.

Remarks 2.8. Assume ${ }_{A} \mathcal{C}$ to be locally projective.

1. $\varpi_{A}: A \otimes_{*_{\mathcal{C}}} \square_{\mathcal{C}} \longrightarrow A$ is surjective iff there exists $\widehat{g} \in \square_{\mathcal{C}}$ with $\widehat{g}(\mathbf{x})=1_{A}$. To prove this assume first that $\varpi_{A}$ is surjective. Then there exist $\left\{\left(a_{i}, g_{i}\right)\right\}_{i=1}^{k} \subset A \times \square \mathcal{C}$, such that $\sum_{i=1}^{k} a_{i} \leftarrow g_{i}=1_{A}$. Set $\widehat{g}:=\sum_{i=1}^{k} a_{i} g_{i} \in \square \mathcal{C}$. Then $\widehat{g}(\mathbf{x})=\left(\sum_{i=1}^{k} a_{i} g_{i}\right)(\mathbf{x})=$ $\sum_{i=1}^{k} g_{i}\left(\mathbf{x} a_{i}\right)=\sum_{i=1}^{k} a_{i} \leftarrow g_{i}=1_{A}$. On the other hand, assume there exists $\widehat{g} \in \square \mathcal{C}$ with $\widehat{g}(\mathbf{x})=1_{A}$. Then for every $a \in A$ we have $1_{A}<(\widehat{g} a)=(\widehat{g} a)(\mathbf{x})=\widehat{g}(\mathbf{x}) a=a$, i.e. $\varpi_{A}$ is surjective.

2. Assume $\varpi_{A}$ to be surjective. If $\Psi_{M}$ is surjective for $M \in \mathcal{M}^{\mathcal{C}}$, then $\varpi_{M}$ is surjective, since

$$
\varpi_{M} \circ\left(\Psi_{M} \otimes_{*_{\mathcal{C}}} i d_{\varpi_{\mathcal{C}}}\right)=\Psi_{M} \circ\left(i d_{M \operatorname{coc}} \otimes_{B} \varpi_{A}\right) .
$$

Theorem 2.9. Assume ${ }_{A} \mathcal{C}$ to be f.g. and projective. Then the following are equivalent:

1. $\mathcal{M}^{\mathcal{C}}$ satisfies the weak structure theorem;

2. ${ }_{B} A$ is flat and $A / B$ is $\mathcal{C}$-Galois;

3. ${ }_{B} A$ is flat and $\beta^{\prime}:=\Psi_{\mathcal{C}}^{\prime}$ is an isomorphism;

4. ${ }_{B} A$ is flat and for every $A$-generated $M \in \mathcal{M}^{\mathcal{C}}=\mathcal{M}{ }^{*} \mathcal{C}$, the $\mathcal{C}$-colinear morphism $\Psi_{M}^{\prime}$ is bijective; 
5. for every $M \in \mathcal{M}^{\mathcal{C}}$, the $\mathcal{C}$-colinear morphism $\Psi_{M}^{\prime}$ is bijective;

6. ${ }_{B} A$ is flat, $\mathcal{M}_{*_{\mathcal{C}}}=\sigma\left[A_{*_{\mathcal{C}}}\right]$ and $\operatorname{Hom}_{-{ }^{*} \mathcal{C}}(A,-): \operatorname{Gen}\left(A *_{\mathcal{C}}\right) \longrightarrow \mathcal{M}_{B}$ is full faithful;

7. $\operatorname{Hom}_{-{ }^{*} \mathcal{C}}(A,-): \mathcal{M}_{*_{\mathcal{C}}} \longrightarrow \mathcal{M}_{B}$ is faithful;

8. $A_{* \mathcal{C}}$ is a generator;

9. $F: Q \otimes_{B} A \longrightarrow{ }^{*} \mathcal{C}$ is surjective (bijective);

10. (a) $Q_{B}$ is f.g. and projective;

(b) $\Omega: A \longrightarrow \operatorname{Hom}_{-B}(Q, B), a \mapsto[q \mapsto a<q]$ is a bimodule isomorphism;

(c) ${ }^{*} \mathrm{C} Q$ is faithful;

11. (a) ${ }_{B} A$ is f.g. and projective;

(b) $\Lambda:{ }^{*} \mathcal{C} \longrightarrow \operatorname{End}\left({ }_{B} A\right)^{o p}, g \mapsto[a \mapsto a<g]$ is a ring isomorphism.

Proof. The result follows by Theorems 1.11, 2.3 and the fact that in case ${ }_{A} \mathcal{C}$ is f.g. and projective $\mathcal{M}^{\mathcal{C}}=\mathcal{M}_{*_{\mathcal{C}}}=\sigma\left[\mathcal{C}_{* \mathcal{C}}\right]$

Theorem 2.10. (Morita, e.g. [Fai81, 4.1.3, 4.3], [MZ97, 2.6]). Let $\mathcal{R}$ be a ring, $P$ a right $\mathcal{R}$-module, $\mathcal{S}:=\operatorname{End}\left(P_{\mathcal{R}}\right)$ and $P^{*}:=\operatorname{Hom}_{\mathcal{R}}(P, \mathcal{R})$.

1. The following are equivalent:

(a) $P_{\mathcal{R}}$ is a generator;

(b) ${ }_{\mathcal{S}} P$ is f.g. projective and $\mathcal{R} \simeq \operatorname{End}\left({ }_{\mathcal{S}} P\right)^{\text {op }}$ canonically.

2. The following are equivalent:

(a) $P_{\mathcal{R}}$ is a faithful quasiprogenerator and ${ }_{\mathcal{S}} P$ is finitely generated;

(b) $P_{\mathcal{R}}$ is a progenerator;

(c) ${ }_{\mathcal{S}} P$ is a progenerator and $P_{\mathcal{R}}$ is faithfully balanced;

(d) $P_{\mathcal{R}}$ and ${ }_{\mathcal{S}} P$ are generators;

(e) $P_{\mathcal{R}}$ and ${ }_{\mathcal{S}} P$ are f.g. and projective;

(f) $\operatorname{Hom}_{-\mathcal{R}}(P,-): \mathcal{M}_{\mathcal{R}} \longrightarrow \mathcal{M}_{\mathcal{S}}$ is an equivalence with inverse $\operatorname{Hom}_{-\mathcal{S}}\left(P^{*},-\right)$;

(g) $-\otimes_{\mathcal{R}} P^{*}: \mathcal{M}_{\mathcal{R}} \longrightarrow \mathcal{M}_{\mathcal{S}}$ is an equivalence with inverse $-\otimes_{\mathcal{S}} P$.

As a consequence of Theorems 2.5] and 2.10 we get

Theorem 2.11. Assume ${ }_{A} \mathcal{C}$ to be f.g. and projective. Then the following are equivalent:

1. $\mathcal{M}^{\mathcal{C}}$ satisfies the strong structure theorem;

2. ${ }_{B} A$ is faithfully flat and $A / B$ is $\mathcal{C}$-Galois; 
3. ${ }_{B} A$ is faithfully flat and $\beta^{\prime}:=\Psi_{\mathcal{C}}^{\prime}$ is bijective;

4. ${ }_{B} A$ is faithfully flat and for every $M \in \sigma\left[A_{*} C\right]$, the map $\Psi_{M}^{\prime}$ is bijective;

5. $A{ }^{* \mathcal{C}}$ is quasiprojective and generates each of its submodules, ${ }_{B} A$ is a weak generator and $\mathcal{M}_{*_{\mathcal{C}}}=\sigma\left[A_{*_{\mathcal{C}}}\right]$

6. $A_{*_{\mathcal{C}}}$ is a quasiprogenerator and $\mathcal{M}_{*_{\mathcal{C}}}=\sigma\left[A_{*_{\mathcal{C}}}\right]$;

7. ${ }_{B} A$ is a weak generator, $\Psi_{M}^{\prime}$ is an isomorphism for every $M \in \operatorname{Gen}\left(A_{*_{\mathcal{C}}}\right)$ and $\mathcal{M}_{*_{\mathcal{C}}}=$ $\sigma\left[A *_{\mathcal{C}}\right]$

8. $A_{*_{\mathcal{C}}}$ is a faithful quasiprogenerator and ${ }_{B} A$ is finitely generated;

9. ${ }_{B} A$ is a progenerator and $A_{*_{\mathcal{C}}}$ is faithfully balanced;

10. $\operatorname{Hom}_{-{ }^{*} \mathcal{C}}(A,-): \mathcal{M}_{* \mathcal{C}} \longrightarrow \mathcal{M}_{B}$ is an equivalence with inverse $\operatorname{Hom}_{-B}(Q,-)$;

11. $-\otimes_{*_{\mathcal{C}}} Q: \mathcal{M}_{*_{\mathcal{C}}} \longrightarrow \mathcal{M}_{B}$ is an equivalence with inverse $-\otimes_{B} A$;

12. $A_{{ }_{*} \mathcal{C}}$ and ${ }_{B} A$ are generators;

13. $A_{*_{\mathcal{C}}}$ and ${ }_{B} A$ are f.g. and projective;

14. $A_{* \mathcal{C}}$ is a progenerator.

\section{Cleft $C$-Galois Extensions}

In what follows $R$ is a commutative ring with $1_{R} \neq 0_{R}$ and $\mathcal{M}_{R}$ is the category of $R$ (bi)modules. For an $R$-coalgebra $\left(C, \Delta_{C}, \varepsilon_{C}\right)$ and an $R$-algebra $\left(A, \mu_{A}, \eta_{A}\right)$ we consider $\left(\operatorname{Hom}_{R}(C, A), \star\right):=\operatorname{Hom}_{R}(C, A)$ as an $R$-algebra with the usual convolution product ( $f \star$ $g)(c):=\sum f\left(c_{1}\right) g\left(c_{2}\right)$ and unity $\eta_{A} \circ \varepsilon_{C}$. The unadorned $-\otimes-$ means $-\otimes_{R}-$.

3.1. Entwined modules. A right-right entwining structure $(A, C, \psi)$ over $R$ consists of an $R$-algebra $\left(A, \mu_{A}, \eta_{A}\right)$, an $R$-coalgebra $\left(C, \Delta_{C}, \varepsilon_{C}\right)$ and an $R$-linear map

$$
\psi: C \otimes_{R} A \longrightarrow A \otimes_{R} C, c \otimes a \mapsto \sum a_{\psi} \otimes c^{\psi},
$$

such that

$$
\begin{aligned}
& \sum(a \widetilde{a})_{\psi} \otimes c^{\psi}=\sum a_{\psi} \widetilde{a}_{\Psi} \otimes c^{\psi \Psi}, \quad \sum\left(1_{A}\right)_{\psi} \otimes c^{\psi}=1_{A} \otimes c, \\
& \sum a_{\psi} \otimes \Delta_{C}\left(c^{\psi}\right)=\sum a_{\psi \Psi} \otimes c_{1}^{\Psi} \otimes c_{2}^{\psi}, \quad \sum a_{\psi} \varepsilon_{C}\left(c^{\psi}\right)=\varepsilon_{C}(c) a .
\end{aligned}
$$

3.2. Let $(A, C, \psi)$ be a right-right entwining structure. An entwined module corresponding to $(A, C, \psi)$ is a right $A$-module, which is also a right $C$-comodule through $\varrho_{M}$, such that

$$
\varrho_{M}(m a)=\sum m_{<0>} a_{\psi} \otimes m_{<1>}^{\psi} \text { for all } m \in M \text { and } a \in A .
$$

The category of right-right entwined modules and $A$-linear $C$-colinear morphisms is denoted by $\mathcal{M}_{A}^{C}(\psi)$. For $M, N \in \mathcal{M}_{A}^{C}(\psi)$ we denote by $\operatorname{Hom}_{A}^{C}(M, N)$ the set of $A$-linear $C$-colinear 
morphisms from $M$ to $N$. With $\#_{\psi}^{o p}(C, A):=\operatorname{Hom}_{R}(C, A)$, we denote the $A$-ring with $(a f)(c)=\sum a_{\psi} f\left(c^{\psi}\right),(f a)(c)=f(c) a$, multiplication $(f \cdot g)(c)=\sum f\left(c_{2}\right)_{\psi} g\left(c_{1}^{\psi}\right)$ and unity $\eta_{A} \circ \varepsilon_{C}$ (see Abu03, Lemma 3.3]).

Entwined modules were introduced by T. Brzeziński and S. Majid [BM98] as a generalization of the Doi-Koppinen modules presented in [Doi92] and [Kop95]. By a remark of M. Takeuchi (e.g. Brz02, Proposition 2.2]), we have an $A$-coring structure on $\mathcal{C}:=A \otimes_{R} C$, where $\mathcal{C}$ is an $A$-bimodule through $a(\widetilde{a} \otimes c):=a \widetilde{a} \otimes c,(\widetilde{a} \otimes c) a:=\sum \widetilde{a} a_{\psi} \otimes c^{\psi}$ and has comultiplication

$$
\Delta_{\mathcal{C}}: A \otimes_{R} C \longrightarrow\left(A \otimes_{R} C\right) \otimes_{A}\left(A \otimes_{R} C\right), a \otimes c \mapsto \sum\left(a \otimes c_{1}\right) \otimes_{A}\left(1_{A} \otimes c_{2}\right)
$$

and counity $\varepsilon_{\mathcal{C}}:=i d_{A} \otimes \varepsilon_{C}$. Moreover $\mathcal{M}_{A}^{C}(\psi) \simeq \mathcal{M}^{\mathcal{C}}, \#_{\psi}^{o p}(C, A) \simeq{ }^{*} \mathcal{C}$ as $A$-rings and ${ }_{A} \mathcal{C}$ is flat (resp. f.g., projective), if ${ }_{R} C$ is so (e.g. Abu03]).

Inspired by [Doi94, 3.1] we make the following definition:

3.3. Let $(A, C, \psi)$ be a right-right entwining structure over $R$ and consider the corresponding $A$-coring $\mathcal{C}:=A \otimes_{R} C$. We say that $(A, C, \psi)$ satisfies the left $\alpha$-condition, if for every right $A$-module $M$ the following map is injective

$$
\alpha_{M}^{\psi}: M \otimes_{R} C \longrightarrow \operatorname{Hom}_{R}\left(\#_{\psi}^{o p}(C, A), M\right), m \otimes c \mapsto[f \mapsto m f(c)]
$$

(equivalently, if ${ }_{A} \mathcal{C}$ is locally projective).

Let $M$ be a right $\#_{\psi}^{o p}(C, A)$-module $M$ and consider the canonical map $\rho_{M}: M \longrightarrow$ $\operatorname{Hom}_{R}\left(\#_{\psi}^{o p}(C, A), M\right)$. Set Rat ${ }^{C}\left(M_{\#_{\psi}^{o p}(C, A)}\right):=\left(\rho_{M}^{\psi}\right)^{-1}\left(M \otimes_{R} C\right)$. We call $M$ \#-rational, if $\operatorname{Rat}^{C}\left(M_{\#^{o p}(C, A)}\right)=M$ and set $\varrho_{M}:=\left(\alpha_{M}^{\psi}\right)^{-1} \circ \rho_{M}$. The category of \#-rational right $\#_{\psi}^{o p}(C, A)$-modules will be denoted by $\operatorname{Rat}^{C}\left(\mathcal{M}_{\#_{\psi}^{o p}(C, A)}\right)$.

Theorem 3.4. (Abu03, Theorem 3.10]) Let $(A, C, \psi)$ be a right-right entwining structure and consider the corresponding $A$-coring $\mathcal{C}:=A \otimes_{R} C$.

1. If ${ }_{R} C$ is flat, then $\mathcal{M}_{A}^{C}(\psi)$ is a Grothendieck category with enough injective objects.

2. If ${ }_{R} C$ is locally projective (resp. f.g. and projective), then

$$
\mathcal{M}_{A}^{C}(\psi) \simeq \operatorname{Rat}^{C}\left(\mathcal{M}_{\#_{\psi}^{o p}(C, A)}\right) \simeq \sigma\left[\left(A \otimes_{R} C\right)_{\#_{\psi}^{o p}(C, A)}\right]\left(\text { resp. } \mathcal{M}_{A}^{C}(\psi) \simeq \mathcal{M}_{\#_{\psi}^{o p}(C, A)}\right) .
$$

In what follows we fix a right-right entwining structure $(A, C, \psi)$ with $\mathcal{C}:=A \otimes_{R} C$ the corresponding $A$-coring and assume that $A \in \mathcal{M}_{A}^{C}(\psi) \simeq \mathcal{M}^{\mathcal{C}}$ with

$$
\varrho_{A}: A \longrightarrow A \otimes_{R} C, a \mapsto \sum a_{<0>} \otimes a_{<1>}=\sum 1_{<0>} a_{\psi} \otimes 1_{<1>}^{\psi} .
$$

Then $\sum 1_{<0>} \otimes 1_{<1>} \in \mathcal{C}$ is a group-like element and

$$
Q \simeq\left\{q \in \operatorname{Hom}_{R}(C, A) \mid \sum q\left(c_{2}\right)_{\psi} \otimes c_{1}^{\psi}=\sum q(c) 1_{<0>} \otimes 1_{<1>} \text { for all } c \in C\right\} .
$$

For every $M \in \mathcal{M}_{A}^{C}(\psi)$, we set

$$
M^{c o \mathcal{C}}:=\left\{m \in M \mid \sum m_{<0>} \otimes m_{<1>}=\sum m 1_{<0>} \otimes 1_{<1>}\right\} .
$$

Moreover we set $B:=A^{c o \mathcal{C}}$. 
Remark 3.5. Let $x \in C$ be a group-like element. For every right $C$-comodule $M$ we put $M^{c o C}:=\left\{m \in M \mid \varrho_{M}(m)=m \otimes x\right\}$. If $\varrho_{A}\left(1_{A}\right)=1_{A} \otimes x$, then we have $M^{c o C}=M^{c o C}$ for every $M \in \mathcal{M}_{A}^{C}(\psi)$.

By [Brz99, Corollaries 3.4, 3.7] $-\otimes_{R}^{c} A: \mathcal{M}^{C} \longrightarrow \mathcal{M}_{A}^{C}(\psi)$ is a functor, which is left adjoint to the forgetful functor. Here, for every $N \in \mathcal{M}^{C}$, we consider the canonical right $A$-module $N \otimes_{R}^{c} A:=N \otimes_{R} A$ with the $C$-coaction $n \otimes a \mapsto \sum n_{<0>} \otimes a_{\psi} \otimes n_{<1>}^{\psi}$.

Proposition 3.6. Let $R$ be a $Q F$ ring and assume $C$ be right semiperfect. Let ${ }_{R} C$ to be locally projective (projective) and put $C^{\square}:=\operatorname{Rat}\left({ }^{*} C C^{*}\right)$.

1. The following are equivalent:

(a) $A$ is a generator in $\mathcal{M}_{A}^{C}(\psi)$;

(b) A generates $C^{\square} \otimes_{R}^{c} A$ in $\mathcal{M}_{A}^{C}(\psi)$;

(c) the map $\Psi_{C^{\square} \otimes_{R}^{c} A}^{\prime}: \operatorname{Hom}_{A}^{C}\left(A, C^{\square} \otimes_{R}^{c} A\right) \otimes_{B} A \longrightarrow C^{\square} \otimes_{R}^{c} A$ is surjective (bijective).

2. The following are equivalent:

(a) $A$ is a progenerator in $\mathcal{M}_{A}^{C}(\psi)$;

(b) $\Psi_{C \square \otimes_{R}^{c} A}^{\prime}$ is surjective (bijective) and ${ }_{B} A$ is a weak generator.

Proof. By [MTW01, 2.6] $C^{\square}$ is a generator in $\mathcal{M}^{C}$, hence $C^{\square} \otimes_{R}^{c} A$ is a generator in $\mathcal{M}_{A}^{C}(\psi)$ by the functorial isomorphism $\operatorname{Hom}_{A}^{C}\left(C^{\square} \otimes_{R}^{c} A, M\right) \simeq \operatorname{Hom}^{C}\left(C^{\square}, M\right)$ for every $M \in \mathcal{M}_{A}^{C}(\psi)$.

1. The assertions follow form the note above and Theorem 2.3.

2. (a) $\Rightarrow$ (b) follows by Theorem 2.5 .

(b) $\Rightarrow$ (a). By the note above $C^{\square} \otimes_{R}^{c} A$ is a generator in $\mathcal{M}_{A}^{C}(\psi)$, and the surjectivity of $\Psi_{C \square \otimes_{R}^{\prime} A}^{\prime}$ makes $A$ a generator in $\mathcal{M}_{A}^{C}(\psi)$. So ${ }_{B} A$ is flat by Theorem 2.3, The weak generator property makes ${ }_{B} A$ faithfully flat and we are done by Theorem 2.5 .

Definition 3.7. A (total) integral for $C$ is a $C$-colinear morphism $\lambda: C \longrightarrow A$ (with $\left.\sum 1_{<0>} \lambda\left(1_{<1>}\right)=1_{A}\right)$. We call the ring extension $A / B$ cleft, if there exists a $\star$-invertible integral. We say $A$ has the right normal basis property, if there exists a left $B$-linear right $C$-colinear isomorphism $A \simeq B \otimes_{R} C$.

Lemma 3.8. Let $\lambda \in \operatorname{Hom}_{R}(C, A)$ be $\star$-invertible with inverse $\bar{\lambda}$. Then:

1. $\lambda \in \operatorname{Hom}^{C}(C, A)$ iff $\bar{\lambda} \in Q$.

2. If $\varrho(a)=\sum a_{\psi} \otimes x^{\psi}$ for some group-like element $x \in C$, then there exists $\widehat{\lambda} \in Q$, such that $\sum 1_{<0>} \widehat{\lambda}\left(1_{<1>}\right)=\widehat{\lambda}(x)=1_{A}$ (in this case $C$ admits a total integral, namely the $\star$-inverse of $\widehat{\lambda})$. 
Proof. Let $\lambda \in \operatorname{Hom}_{R}(C, A)$ be $\star$-invertible with inverse $\bar{\lambda}$.

1. If $\bar{\lambda} \in Q$, then we have for all $c \in C$ :

$$
\begin{aligned}
\sum \lambda\left(c_{1}\right) \otimes c_{2} & =\sum \lambda\left(c_{1}\right) 1_{\psi} \otimes c_{2}^{\psi} \\
& =\sum \lambda\left(c_{1}\right) \varepsilon\left(c_{3}\right) 1_{\psi} \otimes c_{2}^{\psi} \\
& =\sum \lambda\left(c_{1}\right)\left(\bar{\lambda}\left(c_{3}\right) \lambda\left(c_{4}\right)\right)_{\psi} \otimes c_{2}^{\psi} \\
& =\sum \lambda\left(c_{1}\right) \bar{\lambda}\left(c_{3}\right)_{\psi} \lambda\left(c_{4}\right)_{\Psi} \otimes c_{2}^{\psi \Psi} \\
& =\sum \lambda\left(c_{1}\right) \bar{\lambda}\left(c_{22}\right)_{\psi} \lambda\left(c_{3}\right)_{\Psi} \otimes c_{21}^{\psi \Psi} \\
& =\sum \lambda\left(c_{1}\right) \bar{\lambda}\left(c_{2}\right) 1_{<0>} \lambda\left(c_{3}\right)_{\Psi} \otimes 1_{<1>}^{\Psi} \\
& =\sum 1_{<0>} \lambda(c)_{\Psi} \otimes 1_{<1>}^{\Psi} \\
& =\sum \lambda(c)_{<0>} \otimes \lambda(c)_{<1>}
\end{aligned}
$$

i.e. $\lambda \in \operatorname{Hom}^{C}(C, A)$. On the other hand, if $\lambda \in \operatorname{Hom}^{C}(C, A)$, then we have for all $c \in C$ :

$$
\begin{aligned}
\sum \bar{\lambda}\left(c_{2}\right)_{\psi} \otimes c_{1}^{\psi} & =\sum \bar{\lambda}\left(c_{1}\right) \lambda\left(c_{2}\right) \bar{\lambda}\left(c_{4}\right)_{\psi} \otimes c_{3}^{\psi} \\
& =\sum \bar{\lambda}\left(c_{1}\right) \lambda\left(c_{2}\right)_{<0>} \bar{\lambda}\left(c_{3}\right)_{\psi} \otimes \lambda\left(c_{2}\right)_{<1>}^{\psi} \\
& =\sum \bar{\lambda}\left(c_{1}\right) 1_{<0>} \lambda\left(c_{2}\right)_{\psi} \bar{\lambda}\left(c_{3}\right)_{\Psi} \otimes 1_{<1>}^{\psi \Psi} \\
& =\sum \bar{\lambda}\left(c_{1}\right) 1_{<0>}\left(\lambda\left(c_{2}\right) \bar{\lambda}\left(c_{3}\right)\right)_{\psi} \otimes 1_{<1>}^{\psi} \\
& =\sum \bar{\lambda}(c) 1_{<0>} 1_{\psi} \otimes 1_{<1>}^{\psi} \\
& =\sum \bar{\lambda}(c) 1_{<0>} \otimes 1_{<1>},
\end{aligned}
$$

i.e. $\bar{\lambda} \in Q$.

2. Assume $\varrho(a)=\sum a_{\psi} \otimes x^{\psi}$ for some group-like element $x \in C$. Let $\lambda \in \operatorname{Hom}^{C}(C, A)$ with $\bar{\lambda} \in Q$ (see (1)). Then $\hat{\lambda}:=\bar{\lambda} \lambda(x) \in Q$, since $\lambda(x) \in B$, and moreover $\sum 1_{<0>} \widehat{\lambda}\left(1_{<1>}\right)=\widehat{\lambda}(x)=\bar{\lambda}(x) \lambda(x)=(\bar{\lambda} \star \lambda)(x)=\varepsilon_{C}(x) 1_{A}=1_{A}$

Proposition 3.9. Assume $A / B$ to be cleft.

1. $\mathcal{M}_{A}^{C}(\psi)$ satisfies the weak structure theorem (in particular $A / B$ is $\mathcal{C}$-Galois).

2. For every $M \in \mathcal{M}_{A}^{C}(\psi)$, the $C$-colinear morphism

$$
\gamma_{M}: M \longrightarrow M^{c o \mathcal{C}} \otimes_{R} C, m \mapsto \sum m_{<0>} \bar{\lambda} \otimes m_{<1>}
$$

is an isomorphism.

3. A has the right normal basis property.

4. If ${ }_{R} C$ is faithfully flat, then $\mathcal{M}_{A}^{C}(\psi)$ satisfies the strong structure theorem.

Proof. Assume there exists a $\star$-invertible $\lambda \in \operatorname{Hom}^{C}(C, A)$ with inverse $\bar{\lambda} \in Q$ (see Lemma $3.8(1))$. 
1. Let $M \in \mathcal{M}_{A}^{C}(\psi)$ and consider

$$
\widetilde{\Psi}_{M}: M \longrightarrow M^{c o \mathcal{C}} \otimes_{B} A, m \mapsto \sum m_{<0>} \bar{\lambda} \otimes \lambda\left(m_{<1>}\right) .
$$

Then we have for all $n \in M^{c o C}, m \in M$ and $a \in A$ :

$$
\begin{aligned}
\left(\widetilde{\Psi}_{M} \circ \Psi_{M}\right)(n \otimes a) & =\widetilde{\Psi}_{M}(n a) \\
& =\sum\left(n a_{<0>}\right) \bar{\lambda} \otimes_{B} \lambda\left(a_{<1>}\right) \\
& =\sum n a_{<0><0>} \bar{\lambda}\left(a_{<0><1>}\right) \otimes_{B} \lambda\left(a_{<1>}\right) \\
& =\sum n \otimes_{B} a_{<0><0>} \bar{\lambda}\left(a_{<0><1>}\right) \lambda\left(a_{<1>}\right) \\
& =\sum n \otimes_{B} a_{<0>} \bar{\lambda}\left(a_{<1>1}\right) \lambda\left(a_{<1>2}\right) \\
& =n \otimes_{B} a
\end{aligned}
$$

and

$$
\begin{aligned}
\left(\Psi_{M} \circ \widetilde{\Psi}_{M}\right)(m) & =\sum\left(m_{<0>} \bar{\lambda}\right) \lambda\left(m_{<1>}\right) \\
& =\sum m_{<0><0>} \bar{\lambda}\left(m_{<0><1>}\right) \lambda\left(m_{<1>}\right) \\
& =\sum m_{<0>} \bar{\lambda}\left(m_{<1>1}\right) \lambda\left(m_{<1>2}\right) \\
& =\sum m_{<0>} \varepsilon_{C}\left(m_{<1>}\right) 1_{A} \\
& =m .
\end{aligned}
$$

2. For every $M \in \mathcal{M}_{A}^{C}(\psi), \gamma_{M}$ is bijective with inverse

$$
\widetilde{\gamma}_{M}: M^{c o \mathcal{C}} \otimes_{R} C \longrightarrow M, n \otimes c \mapsto n \lambda(c) .
$$

In fact we have for all $m \in M, n \in M^{c o C}$ and $c \in C$ :

$$
\begin{aligned}
\left(\widetilde{\gamma}_{M} \circ \gamma_{M}\right)(m) & =\sum\left(m_{<0>} \bar{\lambda}\right) \lambda\left(m_{<1>}\right) \\
& =\sum m_{<0><0>} \bar{\lambda}\left(m_{<0><1>}\right) \lambda\left(m_{<1>}\right) \\
& =\sum m_{<0>} \bar{\lambda}\left(m_{<1>1}\right) \lambda\left(m_{<1>2}\right) \\
& =\sum m_{<0>} \varepsilon_{C}\left(m_{<1>}\right) \\
& =m
\end{aligned}
$$

and

$$
\begin{aligned}
\left(\gamma_{M} \circ \widetilde{\gamma}_{M}\right)\left(n \otimes_{B} c\right) & =\sum(n \lambda(c))_{<0>} \bar{\lambda} \otimes(n \lambda(c))_{<0>} \\
& =\sum\left(n \lambda(c)_{<0>}\right) \bar{\lambda} \otimes \lambda(c)_{<1>} \\
& =\sum\left(n \lambda\left(c_{1}\right)\right) \otimes c_{2} \\
& =\sum n \lambda\left(c_{1}\right)_{<0>} \bar{\lambda}\left(\lambda\left(c_{1}\right)_{<1>}\right) \otimes c_{2} \\
& =\sum n \lambda\left(c_{11}\right) \bar{\lambda}\left(c_{12}\right) \otimes c_{2} \\
& =n \otimes c .
\end{aligned}
$$

3. By (2) the left $B$-linear right $C$-colinear map

$$
\gamma_{A}: A \longrightarrow B \otimes_{R} C, a \mapsto \sum a_{<0>}<\bar{\lambda} \otimes a_{<1>}
$$

is an isomorphism with inverse $b \otimes c \mapsto b \lambda(c)$.

4. Assume ${ }_{R} C$ to be faithfully flat. By (3) $A \simeq B \otimes{ }_{R} C$ as left $B$-modules, hence ${ }_{B} A$ is faithfully flat. By (1) $A / B$ is $\mathcal{C}$-Glaois and we are done by Theorem 2.5 , 
Theorem 3.10. The following statements are equivalent:

1. A/B is cleft;

2. $\mathcal{M}_{A}^{C}(\psi)$ satisfies the weak structure theorem and $A$ has the right normal basis property;

3. $A / B$ is $\mathcal{C}$-Galois and $A$ has the right normal basis property;

4. $\Lambda: \#_{\psi}^{o p}(C, A) \simeq \operatorname{End}\left({ }_{B} A\right)^{o p}, g \mapsto[a \mapsto a<g]$ is a ring isomorphism and $A$ has the right normal basis property.

If moreover ${ }_{R} C$ is faithfully flat, then (1)-(4) are equivalent to

5. $\mathcal{M}_{A}^{C}(\psi)$ satisfies the strong structure theorem and $A$ has the right normal basis property.

Proof. $(1) \Rightarrow(2)$. This follows by Proposition 3.9 .

(2) $\Rightarrow(3)$. By assumption $\beta:=\Psi_{A \otimes_{R} C}$ is an isomorphism.

(3) $\Rightarrow(4)$. By assumption $A \otimes_{B} A \simeq A \otimes_{R} C$ as left $A$-modules, hence we have the canonical isomorphisms

$$
\begin{aligned}
\#_{\psi}^{o p}(C, A) & \simeq \operatorname{Hom}_{A-}\left(A \otimes_{R} C, A\right) \simeq \operatorname{Hom}_{A-}\left(A \otimes_{B} A, A\right) \\
& \simeq \operatorname{Hom}_{B-}\left(A, \operatorname{End}\left({ }_{A} A\right)\right) \simeq \operatorname{End}\left({ }_{B} A\right) .
\end{aligned}
$$

$(4) \Rightarrow(1)$. Assume $\theta: B \otimes_{R} C \longrightarrow A$ to be a left $B$-linear right $C$-colinear isomorphism and consider the right $C$-colinear morphism $\lambda: C \longrightarrow A, c \mapsto \theta\left(1_{A} \otimes c\right)$ and the left $B$ linear morphism $\delta:=\left(i d \otimes \varepsilon_{C}\right) \circ \theta^{-1}: A \longrightarrow B$. Define $\bar{\lambda}:=\Lambda^{-1}(\delta) \in \#_{\psi}^{o p}(C, A)$. Then we have for all $c \in C$ :

$$
\begin{aligned}
& \sum \lambda\left(c_{1}\right) \bar{\lambda}\left(c_{2}\right)=\sum \lambda(c)_{<0>} \bar{\lambda}\left(\lambda(c)_{<1>}\right) \quad=\quad \lambda(c)<\bar{\lambda} \\
& =\delta(\lambda(c))=\left(\left(i d \otimes \varepsilon_{C}\right) \circ \theta^{-1}\right)(\lambda(c)) \\
& =\left(\left(i d \otimes \varepsilon_{C}\right) \circ \theta^{-1}\right)\left(\theta\left(1_{A} \otimes c\right)\right)=\varepsilon_{C}(c) 1_{A} .
\end{aligned}
$$

On the other hand we have for all $a \in A$ :

$$
\begin{array}{rlrl}
\Lambda(\bar{\lambda} \star \lambda)(a) & =a<(\bar{\lambda} \star \lambda) & & \sum a_{<0>}(\bar{\lambda} \star \lambda)\left(a_{<1>}\right) \\
& =\sum a_{<0>} \bar{\lambda}\left(a_{<1>1}\right) \lambda\left(a_{<1>2}\right) & =\sum a_{<0><0>} \bar{\lambda}\left(a_{<0><1>}\right) \lambda\left(a_{<1>}\right) \\
& =\sum\left(a_{<0>} \leftarrow \bar{\lambda}\right) \lambda\left(a_{<1>}\right) & & =\sum\left(a_{<0>} \leftarrow \Lambda^{-1}(\delta)\right) \lambda\left(a_{<1>}\right) \\
& =\sum \delta\left(a_{<0>}\right) \lambda\left(a_{<1>}\right) & & =\sum \delta\left(a_{<0>}\right) \theta\left(1_{A} \otimes a_{<1>}\right) \\
& =\sum \theta\left(\delta\left(a_{<0>}\right) \otimes a_{<1>}\right) & & =\theta\left(\theta^{-1}(a)\right)=a,
\end{array}
$$

hence $\bar{\lambda} \star \lambda=\eta_{A} \circ \varepsilon_{C}$.

Now assume ${ }_{R} C$ to be faithfully flat. Then (1) $\Rightarrow$ (5) follows by Proposition 3.9 (4) and we are done.

The following result deals with the special case $\varrho(a)=\sum a_{\psi} \otimes x^{\psi}$, for some grouplike element $x \in C$. In this case we obtain the equivalent statements (1)-(5) in Theorem 3.10 without any assumptions on $C$. 
Theorem 3.11. Assume that $\varrho(a)=\sum a_{\psi} \otimes x^{\psi}$ for some group-like element $x \in C$. The following statements are equivalent:

1. $A / B$ is cleft;

2. $\mathcal{M}_{A}^{C}(\psi)$ satisfies the strong structure theorem and $A$ has the right normal basis property;

3. $\mathcal{M}_{A}^{C}(\psi)$ satisfies the weak structure theorem and $A$ has the right normal basis property;

4. $A / B$ is $\mathcal{C}$-Galois and $A$ has the right normal basis property;

5. $\Lambda: \#_{\psi}^{o p}(C, A) \simeq \operatorname{End}\left({ }_{B} A\right)^{o p}, g \mapsto[a \mapsto a<g]$ is a ring isomorphism and $A$ has the right normal basis property.

Proof. By Theorem 3.10 it remains to prove that $\Phi_{N}$ is an isomorphism for every $N \in \mathcal{M}_{B}$, if $A / B$ is cleft. But in our special case there exists by Lemma 3.8 some $\widehat{\lambda} \in Q$ with $\sum 1_{<0>} \widehat{\lambda}\left(1_{<1>}\right)=1_{A}$ and we are done by Corollary $1.8(2)$.

Remark 3.12. Let $(H, A, C)$ be a right-right resp. a left-right Doi-Koppinen structure. Then $(A, C, \psi)$ is a right-right entwining structure with

$$
\psi: C \otimes_{R} A \longrightarrow A \otimes_{R} C, c \otimes a \mapsto \sum a_{<0>} \otimes c a_{<1>}
$$

resp. a left-right entwining structure with

$$
\psi: A \otimes_{R} C \longrightarrow A \otimes_{R} C, a \otimes c \mapsto \sum a_{<0>} \otimes a_{<1>} c
$$

If $x$ is a group-like element of $C$, then $A \in \mathcal{M}(H)_{A}^{C}$ with $\varrho(a):=\sum a_{<0>} \otimes x a_{<1>}$ (resp. $\left.\varrho(a)=\sum a_{<0>} \otimes a_{<1>} x\right)$ and we get [DM92, Theorem 1.5] (resp. [Doi94, Theorem 2.5]) as special cases of Theorem 3.11 .

\section{References}

[Abu03] J.Y. Abuhlail, Rational modules for corings, Commun. Algebra 31, 5793-5840 (2003).

[BDR97] M. Beattie, D. Dăscălescu and Ş. Raianu, Galois extensions for co-Frobenius Hopf algebras, J. Algebra 198, 164-183 (1997).

[BM98] T. Brzeziński and S. Majid, Coalgebra bundles, Comm. Math. Phys. 191, 467492 (1998).

[Brz02] T. Brzeziński, The structure of corings. Induction functors, Maschke-type theorem, and Frobenius and Galois-type properties, Algebr. Represent. Theory 5, 389-410 (2002).

[Brz99] T. Brzeziński, On modules associated to coalgebra Galois extensions, J. Algebra 215, 290-317 (1999). 
[CFM90] M. Cohen, D. Fischman and S. Montgomery, Hopf Galois extensions, smash products and Morita equivalence, J. Algebra 133, 351-372 (1990).

[CMZ02] S. Caenepeel, G. Militaru, and S. Zhu, Frobenius and Separable Functors for Generalized Module Categories and Nonlinear Equations, Lect. Not. Math. 1787, Springer-Verlag, Berlin (2002).

[CS69] S. Chase and M. Sweedler, Hopf algebras and Galois theory, Lec. Not. Math. 97 Springer-Verlag Berlin (1969).

[CVW04] S. Caenepeel, J. Vercruysse and S. Wang, Morita Theory for corings and cleft entwining structures, J. Algebra 276, 210-235 (2004).

[DM92] Y. Doi and A. Masuoka, Generalization of cleft comodule algebras, Comm. Algebra 20, 3703-3721 (1992).

[Doi94] Y. Doi, Generalized smash products and Morita contexts for arbitrary Hopf algebras, Bergen-Montgomery (ed.), Advances in Hopf algebras, Marcle Dekker, Lec. Notes Pure Applied Maths 158, 39-53 (1994).

[Doi92] Y. Doi, Unifying Hopf modules, J. Algebra 153, 373-385 (1992).

[Doi83] Y. Doi, On the structure of relative Hopf modules, Comm. Algebra 11(3), 243-255 (1983).

[DT89] Y. Doi and M. Takeuchi, Hopf-Galois extensions of algebras, the MiyashitaUlbrich action, and the Azumaya algebras, J. Algebra, 121, 488-516 (1989).

[DT86] Y. Doi and M. Takeuchi, Cleft comodule algebras for a bialgebra, Comm. Algebra 14, 801-817 (1986).

[Fai81] C. Faith, Algebra I, Rings, Modules and Categories, Springer-Verlag (1981).

[Kop95] M. Koppinen, Variations on the smash product with applications to groupgraded rings, J. Pure Appl. Algebra 104, 61-80 (1995).

[KT81] H. Kreimer and M. Takeuchi, Hopf Algebras and Galois extensions of an algebra, Indiana Univ. Math. J. 30, 675-692 (1981).

[MSTW01] C. Menini, A. Seidel, B. Torrecillas and R. Wisbauer, A-H-bimodules and equivalences, Comm. Algebra 29(10), 4619-4640 (2001).

[MTW01] C. Menini, B. Torrecillas and R. Wisbauer, Strongly rational comodules and semiperfect Hopf algebras over QF Rings, J. Pure Appl. Algebra 155, 237-255 (2001).

[MZ97] C. Menini and M. Zuccoli, Equivalence theorems and Hopf-Galois extensions, J. Algebra 194, 245-274 (1997).

[Sch90] H-J. Schneider, Principal homogenous spaces for arbitrary Hopf algebras, Israel J. Math. 72(1-2) 167-195 (1990). 
[Swe75] M. Sweedler, The Predual theorem to the Jacobson-Bourbaki theorem, Trans. Amer. Math. Soc. 213, 391-406 (1975).

[Wis02] R. Wisbauer, On the category of comodules for corings, Proc. 3rd. Int. Pal. Conf.: Math. \& Math. Edu., Bethlehem (Palestine), S. Elaydi et al. (ed.), World Scientific, New Jersy, ISBN 981-02-4720-6, 325-336 (2002).

[Wis88] R. Wisbauer, Grundlagen der Modul- und Ringtheorie, München: Verlag Reinhard Fischer (1988); Foundations of Module and Ring Theory, Gordon and Breach, Reading (1991).

[Z-H76] B. Zimmermann-Huignes, Pure submodules of direct products of free modules, Math. Ann. 224, 233-245 (1976). 